\title{
Lifestyle, Physical and Cardiovascular Components Associated with Immune Profile in Hispanic-Latino People Living with HIV
}

Martín G. Rosario PT, Ph.D., CSFI, ATRIC ${ }^{*}$, Gabriel Gines BS, MPH, CSFI, CPT ${ }^{2}$, Leah Jamison DPT ${ }^{1}$

${ }^{1}$ Physical Therapy Program, Texas Woman's University, Dallas Campus, United States.

${ }^{2}$ Ponce Health Science University School, United States.

Article Details
Article Type: Research Article
Received date: $23^{\text {rd }}$ June, 2020
Accepted date: $27^{\text {th }}$ July, 2020
Published date: $29^{\text {th }}$ July, 2020

*Corresponding Author: Martín G. Rosario PT, Ph.D., CSFI, ATRIC, Physical Therapy Program, Texas Woman's University, Dallas Campus; 5500 Southwestern Medical Ave. Dallas, TX 75235-7299, United States. E-mail: mrosario1@twu.edu Citation: Rosario, M.G., Gines, G., Jamison, L. (2020). Lifestyle, Physical and Cardiovascular Components Associated with Immune Profile in Hispanic-Latino People Living with HIV. J Ment Health Soc Behav 2(1):121. https://doi.org/10.33790/ jmhsb1100121

Copyright: (C2020, This is an open-access article distributed under the terms of the Creative Commons Attribution License 4.0, which permits unrestricted use, distribution, and reproduction in any medium, provided the original author and source are credited.

\section{Abstract}

HIV is associated with poor quality of life and high mortality rates on the people suffering from this illness. Presently, Cd4 levels are the main factor established to identify the distinct trauma on the host's body and systems. The consequence and severity of HIV complications vary among individuals; however, many factors come into play when assessing and developing treatment plans for this population.

Purpose: The current investigation endeavors to respond to the following, are there any indicators of a weak immune system other than a low Cd4 count in people living with HIV (PLHIV) that can aid and guide more target treatment to increase life expectancy and improve quality of life? This project's invention is to establish a comprehensive profile equated with the immune system levels among Hispanics-Latino PLHIV.

Methods: Data was extracted and collected from 449 partakers' records from participants enrolled in La Perla de Gran Precio HIV Community Center in Puerto Rico. We examined and compared the relationship between five distinct components, motor (speed and inclination on a treadmill), cardiorespiratory (cardio time and HR), lipid panel (triglycerides, cholesterol among others), comorbidities (diabetes, neuropathy, among others) and lifestyle (smoker, sedentary, etc.), which are essential physical fitness components, regarding subjects' immune profiles (Cd4 values).

Results: The data analyzed in this study were from 449 participants' records further divided into 198 for the Low-HIV $(\mathrm{Cd} 4=323.6+/$ 120.75) and 247 for the High-HIV (Cd4 $=836.84+/-283.41)$ groups according to $\mathrm{Cd} 4$ values. The L-HIV and H-HIV were, for the most part, males with an average age of 54 years old and approximately 20 years of diagnosis. An ANOVA analysis revealed that four out of the five components showed differences among both groups.

Conclusion: In Hispanic Latino PLHIV, distinctive physical and cardiovascular profiles, related to lower immune health, were identified. We recommend adding the components mentioned in this study to comprehensively understand the mechanism affecting the quality of life and life expectancy in those with HIV.

\section{Introduction}

The human immunodeficiency virus (HIV) affects 36.9 million people worldwide, 1.1 million of which are in the United States (US) alone. Within the US, Texas and Puerto Rico are among the areas with the most substantial incidence rates, which have increased in the last few years [1]. Primarily in the US, Hispanic Latino males are among the group of individuals and ethnicity vastly affected by this disease. HIV is a disease that assaults the brain, eliciting alterations that ultimately affects the cognitive and motor components of the people living with this virus.

The disruptions created by HIV, in both motor and cognitive elements, interfere with ordinary activities and are associated with a poor quality of life. These alterations caused by the virus are valuable to point out since people living with HIV (PLHIV) over the age of 50 years has a higher endangerment of falls, injuries, and balance alterations compared to people without the disease in the same age group [2]. Antiretroviral therapy (ART) has managed to increase life expectancy and reduce the negative consequence created by the virus; however, not without side effects. The longer PLHIV lives, the higher the period, and opportunities for additional complications affecting the quality of life in these individuals.

HIV infection, when untreated, replicates in the body's hosts weakening the immune system. When the immune system is vulnerable, the body is unable to fight infections or diseases, creating a bombardment of every system that eventually will kill the host. Without treatment HIV advances into three stages according to CDC standards (www.cdc.gov); stage one: Acute HIV infection, stage two: Chronic HIV infection, and stage three: AIDs. Stage one, PLHIV can range from being asymptomatic to experiencing life threatening symptoms. During this stage large amounts of the virus are being produced, Cd4 cells fall rapidly, and are at the highest risk of transmitting the virus to others. Stage two, PLHIV that is on ART can live with clinical latency for several decades as the treatment keeps the virus in a safe zone. PLHIV that do not take ART decrease their mortality rate and only last approximately 10 years before advancing to stage 3, AIDs. Nowadays, ART is utilized to prevent or reduce the detrimental effects caused by this disease.

However, despite advances in ART, HIV still detrimentally affects cardiovascular system and motor aspects of gait and balance, steering to an increased risk of falls, injury, poor quality of life and early mortality [3-5]. The characteristics affecting the quality of life, provoking a premature death in PLHIV have gained much importance in the last several years. For instance, reports on body composition for PLHIV illustrates the association between obesity with a subnormal performance during dynamic activities, including 
walking [6]. PLHIV with an obese body mass exhibited significantly more gait and balance alterations compared to PLHIV with underweight or frail composition [7]. For those living with HIV, the statement mentioned above is a problem. Not only specific explanations regarding the association with obesity and motor deficiencies in PLHIV are unknown, but also one of the many side effects of ART is hyperlipidemia, a condition that changes the body composition, causing weight gain and obesity in these people.

Various studies highlight the clinical implication of body composition and cardiovascular fitness [8,9]. Cardiovascular exercise from moderate (walking) to intense (jogging) has been proven to be beneficial to PLHV [10]. Contemplating the above, cardiovascular assessments are advised and a necessity for all PLHIV [11]. Several researchers study and describe the many benefits of cardiovascular fitness in PLHIV. For instance, in PLHIV, cardiovascular exercise alone has proven to be not only safe but also to share the same cardiorespiratory advantages as to HIV negative individuals [12]. Improvements in quality of life (QoL) [13-16], ADLs [17], stress reduction [18], and mood [19] are among the benefits associated with cardiovascular fitness.

Over the years, researchers studied the benefits of the combination of cardiovascular exercise with adjunct modalities. Few of these modalities include diet to reduce lipid levels [20], nutrition to improve QoL [21] and progressive resistance exercise (PRE) with flexibility exercises [22]. The ultimate goal is to improve QoL by promoting independence in PLHIV.

One significant benefit of cardiovascular fitness is a healthy immune system. However, there are conflicts on the benefits of cardiovascular exercises and the role of the immune systems in PLHIV. Various authors reported advantages of cardiovascular profile on boosting the $\mathrm{Cd} 4$. Others stated a consistency on the $\mathrm{Cd} 4$ values of their participants.

$\mathrm{Cd} 4$ count is the gold standard for the assessment of the immune system in PLHIV. The higher the Cd4 value, the healthier the person, and the more effective ART is on the body/system. There are various HIV classifications according to the $\mathrm{Cd} 4$ count that categorize the immune system profile, how healthy or affected, are the individuals [23]. This work is asking, could the cardiovascular fitness profile be different depending on the $\mathrm{Cd} 4$ values and HIV classification? Could the physical profile be more affected when Cd4 values are at lower levels? And finally, are there any indicators of a weak immune system other than a low Cd4 count in PLHIV?

With that intent, this work aims to investigate the impact of different physical and fitness profiles on health, wellness, and disease progression among PLHIV to varying stages of the disease. The Cd4 count is a measurement utilized by clinicians treating PLHIV in every medical field to assess the extent of the patients' disease. HIV is diagnosed in people with a Cd4 count above 200, whereas AIDS is categorized as having a Cd4 count below 200. To develop targeted treatments within the constraints of the disease, investigating the differing fitness profiles of PLHIV in correlation to their Cd4 count is imperative. We suggest that having a specific distinction among the $\mathrm{Cd} 4$ will aid, halt, or delay the consequences related to HIV. In pursuit of investigating this distinction, this inquiry examines the relationship between the motor (speed and inclination on a treadmill), cardiorespiratory (cardio time and HR), lipid panel (triglycerides, cholesterol among others), comorbidities (diabetes, neuropathy among others) and lifestyle (smoker, sedentary, among others) values, which are prime physical fitness components, regarding subjects' immune profiles (Cd4 values).

We hypothesize that individuals with low Cd4 (less than 500) will exhibit a reduced motor and cardiovascular component, with higher occurrence of comorbidities and inappropriate lifestyle choices compared to subjects with higher $\mathrm{Cd} 4$ values (501 and above).

\section{Methods}

Participants were enlisted into a Community Based Center (La Perla de Gran Precio) in San Juan Puerto Rico from 2000-2020. All partakers were cleared to participate in the community center by their primary doctor responsible for the follow-ups and progression of the HIV conditions. The main focus of the La Perla de Gran Precio (LPGP) community center is wellness and improvement of quality of life through exercise therapy to Hispanic Latino PLHIV.

Therefore, participants were assessed by a Physical Therapist prior any further involvement in the fitness center. Then, a Certified Personal Trainer performs all the exercise testing, including the cardiovascular test reported in this test, the submaximal Ross treadmill test.

A critical aspect to point out is that the current work collected retrospective data from participants' records from the last 20 years. This study and work was approved by the LPDG and followed the privacy and confidentiality standard established by the said establishment.

As part of the LPDG requirements, all participants bring their current lab work and perform a series of fitness tests. The cardiovascular and motor components were collected with the Ross submaximal cardiovascular test. The lifestyle, comorbidities, immune (Cd4) and lipid panel information was collected by interview and the latest blood work.

\section{Motor and Cardiovascular components}

Prior to the cardiovascular test, the vitals of participants were collected. The Ross treadmill test started with participants walking at a $2.0 \mathrm{mph}$ speed with 0 inclination. The first six minutes of the test consisted in speed and inclination. At minute 2 speed increased to $2.5 \mathrm{mph}$, at minute 3 speed increased to 3.0 and at minute 4 speed increased to $3.4 \mathrm{mph}$ with zero inclination. With a constant speed of $3.4 \mathrm{mph}$ the inclination increased $3 \%$ every 3 minutes, for a maximal inclination of $15 \%$ at 21 minutes. The test stopped when maximal cardiac frequency was achieved or when participants reported the onset of muscle or cardiovascular fatigue. The cardiovascular data reported in this study were the values at the moment the Ross test ended. Data retrieved from participants' records were heart rate and blood pressure for cardiovascular components and speed and inclination of the treadmill for the motor components.

\section{Lifestyle, comorbidities, lipids and immune components}

During the initial or follow-ups interviews, participants reported lifestyle information such as being a smoker or sedentary as well as currently diagnosed comorbidities (diabetes, high blood pressure along with others). Part of the above information in addition to the lipid and Cd4 labs were collected from the blood work and doctor notes or scripts.

\section{The unique data collection and database development was as} follows

1. Data from all participants enrolled in La Perla de Gran Precio (LPGP) from 2000-2020, who had finalized the Center's enrollment prerequisite (approximately 1,300 files), were scrutinized for data/information and completeness. LPGP participants underwent longitudinal assessments, including, but not limited to, documentation of comorbidities, medications, lipid panel, years with HIV diagnosis, demographics (age, gender), HIV status (Cd4), vitals and physical activity status. This study recorded the most recent information available in the records for all participants. Most of the participants of this HIV clinic are Hispanic/Latino (in the United States); thus, this project will give us a favorable cardiovascular profile of their ethnic group.

2. After the data collection, a codebook was prepared for data mapping and comparisons. The codebook also provides data code 
and variable definition. Then, the data was organized in a database for further analysis, comparison, and interpretation.

3. Data was divided according to $\mathrm{Cd} 4$ values. Participants with a Cd4 count from 500 or below were allocated in one group, low Cd4 group (L-HIV group) and subjects with Cd4 of 501 or higher were assigned to the high $\mathrm{Cd} 4$ group (H-HIV group) for comparison.

\section{Data Analysis}

This study performed an analysis of variance (ANOVA) with SPSS for the comparison of both groups. Five components were collected and compared, cardiovascular, comorbidities, motor, lifestyle and lipid profile. Motor components included the speed and inclination of the treadmill as values of interest and the cardiorespiratory components utilized time of test completion and vitals (heart rate and blood pressure) at the moment that the Ross test ended.

\section{Results}

To ascertain the association mentioned above, to physical and fitness outlines and the immune profile, this work divides $\mathrm{Cd} 4$ counts into two ranges: 1) 0-500, and 2) 501-above.

Table 1 depicts the demographic outlines of the participants in the current study. The examined data in this investigation were collected from 449 participants' records, and further divided into 198 for the L-HIV (Cd4=323.6+/- 120.75) and 247 for the H-HIV $(\mathrm{Cd} 4=836.84+/-283.41)$ groups according to $\mathrm{Cd} 4$ values. The L-HIV and H-HIV were comparable in age (L-HIV $=54+/ 9.73$ and H-HIV $54.42+/ 10.16$ year), years of diagnosis $(\mathrm{L}-\mathrm{HIV}=20.98+/-8.73$ and $\mathrm{H}-\mathrm{HIV}=19.84+/-8.42)$ and gender ratio, being mostly males.

\begin{tabular}{|l|l|l|l|}
\hline Characteristics & $\begin{array}{l}\text { Low Cd4 } \\
\mathbf{n}=\mathbf{1 9 8}\end{array}$ & $\begin{array}{l}\text { High Cd4 } \\
\mathbf{n}=\mathbf{2 5 1}\end{array}$ & P value \\
\hline Age & $\mathrm{M}=54+/ 9.73$ years & $\mathrm{M}=54.42+/ 10.16$ years & $\mathrm{P}=.673$ \\
\hline Gender & Male= 168 & $\begin{array}{l}\text { Male= 198 } \\
\text { Female }=30\end{array}$ & $\mathrm{Female}=53$ \\
& $\mathrm{M}=0.26+/-0.441$ & $\mathrm{M}=0.29+/-0.457$ & \\
\hline Year of Dx & $\mathrm{M}=20.98+/-8.73$ & $\mathrm{M}=836.84+/-8.42$ & $\mathrm{P}=.235$ \\
\hline Cd4 & $\mathrm{M}=323.6+/-120.75$ & $\mathrm{P}=.001$ \\
\hline
\end{tabular}

\section{Cardiovascular and Motor Components}

Table 2A portrays the equivalence of the cardiovascular factors between groups. The L-HIV group exhibits an increase $(\mathrm{P}<0.05)$ in heart rate compared to the H-HIV group. The other appraised cardiovascular components were comparable between groups $(\mathrm{P}>0.005)$. The motor component portrayed by the gait velocity and treadmill inclination (Table $2 \mathrm{~A}$ ) were analogous among groups $(\mathrm{P}>0.005)$.

\begin{tabular}{|l|l|l|l|l|}
\hline Characteristics & $\begin{array}{l}\text { Low Cd4 } \\
\mathbf{n}=\mathbf{1 9 8}\end{array}$ & $\begin{array}{l}\text { High Cd4 } \\
\mathbf{n}=\mathbf{2 5 1}\end{array}$ & F Value & P value \\
\hline Heart Rate (bpm) & $\mathrm{M}=140.04+/-18.55$ & $\mathrm{M}=135.63+/-19.04$ & 3.94 & $\mathrm{P}=.05$ \\
\hline Systolic BP $(\mathrm{mmHg})$ & $\mathrm{M}=122.95+/-17.35$ & $\mathrm{M}=125.24+/-16.33$ & 0.659 & $\mathrm{P}=.222$ \\
\hline $\begin{array}{l}\text { Diastolic BP } \\
(\mathrm{mmHg})\end{array}$ & $\mathrm{M}=74.98+/ 10.13$ & $\mathrm{M}=75.41+/-10.83$ & 0.005 & $\mathrm{P}=.717$ \\
\hline Cardio test Time & $\mathrm{M}=10.49+/-4.83$ & $\mathrm{M}=10.03+/-4.57$ & .048 & $\mathrm{P}=.321$ \\
\hline Motor Component at the end of the Ross Submaximal Test & $\mathrm{P}=.915$ \\
\hline Velocity & $\mathrm{M}=3.31+/-.273$ & $\mathrm{M}=3.31+/-.270$ & .985 & $\mathrm{P}=.196$ \\
\hline Inclination & $\mathrm{M}=5.71+/-4.33$ & $\mathrm{M}=5.18+/-4.03$ & .090 & \\
\hline
\end{tabular}

\section{Comorbidity, Lipid, and Lifestyle Components}

Among the 10 comorbidities listed on Table 2B; high blood pressure, neuropathy, and hyperlipidemia were reported significantly more in the H-HIV group than the L-HIV group. The lipid component (Table 2B) exemplifies the specific lipid values of all participants. Results showed a considerable increase in cholesterol and HDL values $(\mathrm{P}<0.05)$ in the H-HIV group when compared to L-HIV. For the lifestyle component, Table $2 \mathrm{~B}$ displays the main comorbidities documented by all participants. Among the five listed comorbidities, partakers in the L-HIV reported smoking significantly $(\mathrm{P}<0.005)$ more when compared to H-HIV. The additional assessed aspects of the comorbidity components were compared among groups; however, the H-HIV group reported having significantly more comorbidities than the counterpart. The number of ART medications was similar among these groups.

\section{Discussion}

This inquiry had the intention of exploring and describing the different physical fitness profiles on health, wellness, and disease progression in Hispanic Latino people. In pursuit of investigating this distinction, this inquiry explored the relationship between the motor (speed and inclination on a treadmill), cardiorespiratory (cardio time and HR), lipid panel (triglycerides, cholesterol among others), comorbidities (diabetes, neuropathy among others) and lifestyle (smoker, sedentary, among other variables, which are essential physical fitness components, regarding subjects' immune profiles (Cd4 values) in Hispanic Latinos living with HIV. To verify the association referred above, to physical fitness profiles and the immune profile, this endeavor distributes $\mathrm{Cd} 4$ counts into two, low ranges: 0-500, and high range 501-above of $\mathrm{Cd} 4$.

To that effect, this endeavor attempted to answer the following questions. Foremost, could the cardiovascular fitness profile be distinct depending on the $\mathrm{Cd} 4$ values and HIV classification? The cardiovascular values were, for the most part, related among groups, except for heart rate, which was lower in the H-HIV group. Our findings propose employing heart rate as a criterion for cardiovascular assessment like previously reported. 


\begin{tabular}{|c|c|c|c|c|}
\hline Characteristics & Low Cd4 n=198 & High Cd4 n=251 & F Value & P value \\
\hline High Blood Pressure & $\mathrm{M}=0.18+/ 0.383$ & $\mathrm{M}=0.31+/-0.465$ & 7.07 & 0.01 \\
\hline Chronic Pain & $\mathrm{M}=.09+/-0.291$ & $\mathrm{M}=0.17+/-0.375$ & 3.45 & 0.06 \\
\hline Neuropathy & $\mathrm{M}=0.13+/-0.333$ & $\mathrm{M}=0.30+/-0.458$ & 12.05 & 0.001 \\
\hline Depression & $\mathrm{M}=0.27+/-0.445$ & $\mathrm{M}=0.22+/-0.416$ & 0.88 & 0.35 \\
\hline Diarrhea & $\mathrm{M}=.03+/-0.181$ & $\mathrm{M}=.03+/-0.184$ & 0.00 & 0.95 \\
\hline Hiperlipidemia & $\mathrm{M}=0.15+/-0.360$ & $\mathrm{M}=0.26+/-0.438$ & 4.62 & 0.05 \\
\hline Arthritis & $\mathrm{M}=0.01+/-.092$ & $\mathrm{M}=.05+/-0.211$ & 3.42 & 0.07 \\
\hline Anemia & $\mathrm{M}=0.03+/-0.181$ & $\mathrm{M}=0.02+/-0.151$ & 0.28 & 0.60 \\
\hline Diabetes & $\mathrm{M}=0.05+/-0.220$ & $\mathrm{M}=0.08+/-0.274$ & 1.05 & 0.31 \\
\hline Others Dx & $\mathrm{M}=0.20+/-0.403$ & $\mathrm{M}=0.20+/-0.405$ & 0.00 & 0.95 \\
\hline \multicolumn{5}{|c|}{ Lipids Components Reported in the Lab analysis } \\
\hline Characteristics & Low Cd4 n=198 & High Cd4 n=251 & F value & P value \\
\hline Cholesterol & $\mathrm{M}=165.63+/-38.38$ & $\mathrm{M}=184.19+/-47.51$ & 8.43 & 0.01 \\
\hline Triglyceride & $\mathrm{M}=164.47+/-85.69$ & $\mathrm{M}=187.45+/-158.66$ & 1.28 & 0.26 \\
\hline HDL & $\mathrm{M}=41.59+/-11.33$ & $\mathrm{M}=47.17+/-19.94$ & 4.24 & 0.05 \\
\hline VLDL & $\mathrm{M}=33.38+/-12.32$ & $\mathrm{M}=41.85+/-38.06$ & 1.53 & 0.22 \\
\hline $\mathrm{CHOL} / \mathrm{HDL}$ & $\mathrm{M}=7.63+/-24.16$ & $\mathrm{M}=4.23+/-1.53$ & 1.46 & 0.23 \\
\hline \multicolumn{5}{|c|}{ Lifestyle Components Reported by Participants } \\
\hline Characteristics & Low Cd4 n=198 & High Cd4 n=251 & F value & Pvalue \\
\hline Over 50 years & $\mathrm{M}=0.18+/-0.39$ & $\mathrm{M}=0.26+/-0.44$ & 2.34 & 0.13 \\
\hline Stress & $\mathrm{M}=0.18+/-0.39$ & $\mathrm{M}=0.26+/-0.44$ & 3.23 & 0.07 \\
\hline Smoker & $\mathrm{M}=0.28+/-0.45$ & $\mathrm{M}=0.16+/-0.37$ & 6.31 & 0.01 \\
\hline Obese & $\mathrm{M}=0.11+/-0.31$ & $\mathrm{M}=0.15+/-0.36$ & 1.06 & 0.30 \\
\hline Sedentary & $\mathrm{M}=0.17+/-0.38$ & $\mathrm{M}=0.12+/-0.33$ & 1.22 & 0.27 \\
\hline MC-Multiple Comorbidities & $\mathrm{M}=1.07+/-1.14$ & $\mathrm{M}=1.38+/-1.38$ & 4.12 & 0.05 \\
\hline Meds- Multiple Meds & $\mathrm{M}=2.19+/-1.04$ & $\mathrm{M}=1.99+/-1.04$ & 2.25 & 0.14 \\
\hline
\end{tabular}

Another query this study aimed to answer is, is there a variation in the physical profile when $\mathrm{Cd} 4$ values are at downward levels? Four out of the five components reviewed in this study depicted distinctions with $\mathrm{Cd} 4$ fluctuations. Our study proposes adding some of these elements to the screening process of PLHIV.

The final question we asked was, can indicators pertain to a physical and cardiovascular profile aid in identifying an unstable immune system further than a low Cd4 count in PLHIV? This investigation recognized various indicators entailing lipids, heart rate, and inadequate lifestyle habits, still, failed to discover any motor factors associated with $\mathrm{Cd} 4$ variations. Although a more thorough correlation is integral, various of our variables reveal tendencies and connections to immune system variations. Deeming all the traits reflected above, we hypothesized that individuals with low Cd4 (less than 500) would exhibit a lessening of motor and cardiovascular components, with a higher occurrence of comorbidities and inadequate lifestyle preferences distinguished those with higher $\mathrm{Cd} 4$ values (501 and above). With the findings and tendencies identified in the current study, we partially accept our hypothesis.

\section{Cardiovascular Component}

Our study demonstrates the cardiovascular components represented by the heart rate variables are compensating differently when $\mathrm{Cd} 4$ values are lower than 500. Previous studies postulate the implication of cardiovascular training on cardiopulmonary health, mood [24], participation in activities of daily living [17], and quality of life [15] among others in PLHIV. The Ross test measures the cardiovascular response in a total of 21 minutes, the average of test completion for the participants in this study was approximately 10 minutes for both groups, suggesting a compromised or unhealthy cardiopulmonary system. Various researches focusing on the cardiovascular component of PLHIV established the association of HIV disease and cardiovascular dysfunction, particularly diastolic dysfunction [25]. When the virus is untreated, with a low Cd4 immune system as well as aids diagnosis, the prevalence of cardiac complications is higher. Although with ART medication, the prevalence and incidence have reduced significantly, heart failure is an existing issue among PLHIV [26] even with no history of cardiovascular disease [25]. Our findings demonstrate continuing utilizing target heart rate to tailored cardiopulmonary intervention programs during all stages of the disease. Similar to previous authors, we recommend cardiopulmonary assessment in all stages of the disease, to reduce the occurrence of cardiovascular complications in PLHIV [26, 27].

\section{Motor Component}

PLHIV exhibited neuromotor alterations in the form of gait and balance difficulties. The current study proves the similarities in the motor aspects of the treadmill Ross test. Although the speed and treadmill inclination were comparable in both groups, both parameters were lower than the standards of the test, therefore, equally affected. Treadmill inclination mimics real ground walking, accordingly, the difficulty increases with the increment in angle of inclination [28]. Studies focusing on the motor components of balance highlights the deficiencies of postural instability and neuromuscular modification in asymptomatic PLHIV [29]. Balance alterations caused by the HIV disease can potentially affect the quality of life in this population. Currently, there are portions of information missing to properly aid on the identification of the mechanism affecting motor control on PLHIV, such as the gait profile. 
We encourage including gait training with challenging elements such as uneven surface, hills, ramps, and any aspects that engage lower limb musculature and the cardiorespiratory system, regardless of the stage of the HIV disease.

\section{Comorbidity Component}

The current investigation exemplifies the most common comorbidities (high blood pressure, neuropathy, and hyperlipidemia) specific to differences cd4 score in Hispanic Latino PLHIV. To our marvel, all three conditions were more prevalent in the H-HIV group with a less compromised immune system. Rosario et al. [29] highlighted depression followed by high blood pressure, as the most common comorbidities in the Hispanic Latino PLHIV. Contrary to statements related to frontal lobe alterations and depression suffered by PLHIV, our current study found an additional issue associated with this illness. Our results suggest that regardless of the cd4 values, due to the HIV disease or the ART medications, both groups displayed comorbidities; however, withstood at a higher rate by the H-HIV group. Previous studies identified a link between several ART medications and hyperlipidemia accompanied with the urgency of pharmacological therapy to reduce the effects on health in PLHIV $[30,31]$. One aspect of the outcomes we seek to accentuate is the knowledge that the group with the higher $\mathrm{Cd} 4$ count had more comorbidities compared to the L-HIV, and one of the afflictions was peripheral neuropathy (PN). PN is connected to muscle activation difficulties leading to postural instability in those suffering from this illness [32]. Distinct to PLHIV, the association of PN with the HIV disease and ART has been cited in previous research among Latino Hispanic individuals [29]. Like those above, we advise cardiopulmonary inspection and intervention in addition to $\mathrm{PN}$ appraisal, comprising monofilament for the diagnosis of PN and treatment in all PLHIV. In the future, inquiries correlating immune system phases, years living with HIV infection, as well as ART and PN medication, are suggested.

\section{Lipid Component}

This study highlights some risk factors associated with the H-HIV group, higher cholesterol, and HDL. Our results denote the importance of routine lipid panel lab work regardless of the cd4 values. Since the implementation of ART to treat HIV, hyperlipidemia has been a burden and a conceivable complication to PLHIV. Earlier studies reveal the urgency of eliminating reversible risk factors such as smoking and sedentary life to avoid further health complications, like coronary artery disease [33]. Medications to treat hyperlipidemia are useful in combination with lifestyle modifications like boosting cardiopulmonary fitness, favorable diet, and quitting smoking [31]. As the ART medication cocktails evolves more effectively in lengthening life expectancy in PLHIV, numerous lifestyle modifications should be incorporated. The current study and outcomes reflected the association between cd4 values and a lipid profile, making noticeable that regardless of the $\mathrm{Cd} 4$ number (high or low cd4), hyperlipidemia is a likelihood, followed by the increased risk of coronary artery disease, which is an issue worth assessing in PLHIV in all stages of the disease. Our study further recommends discerning body composition (for instance, body mass index) to trace body fat distribution across the participant's life. The aforementioned is one crucial limitation of this study. Since data collection was acquired through participant's records, not an interview, body composition measurements were not compiled in more than $75 \%$ of the interviews, thus currently missing from the participant's history; therefore, we were unable to acquire this piece of information.

\section{Lifestyle Component}

This study highlights the distinctions between high and low immune system status. Among the variations attributed to lifestyle, smoking was documented considerably more in the L-HIV. Outcomes of this study evoke that smoking might be associated with the lower levels of cd4 and not the quantity of ART intake by these participants. Reported reasons are explaining the lower Cd4 values in these PLHIV. Among the assumptions, inconsistency with ART medication, the onset of ART therapy compared to the timeframe of contracting the virus, and lifestyle choices [34] are among the reasons Cd4 might be variable in the present study. Within the lifestyle choice and as previously mentioned, smoking was the recurrent factor among the groups in this study. Smoking has been associated with depression in PLHIV. It is reported in the literature as a critical factor for health-related quality of life deteriorating, life expectancy reduction, and qualityadjusted life expectancy alterations [35]. In the current work, the outcomes presented in this study we collected from participants' records and not with interviews, as previously mentioned. As such, limitations on the type of specific data collected were limited; for instance, time of infection with the virus, the onset of ART initiation and adherence, mortality rates, and life expectancy were not captured in the participants' records or the present study. Therefore, for a more comprehensive assessment of the impact of lifestyle choices among Hispanic-Latinos living with HIV, we suggest collecting data such as time of diagnosis, the onset of ART, history of ART, ART adherence, and mortality information.

\section{Conclusion}

The present study ushers on a unique comparison of physical and cardiovascular components associated with the immune health of Hispanic Latino PLHIV. Various distinctions were illustrated in this study related to the $\mathrm{Cd} 4$ levels, except for the motor component, which was similar among groups. Lipid profile, poor lifestyle choices, and cardiopulmonary components are among the factors that might be affecting the quality of life in this particular group, therefore essential to assess in PLHIV. We encourage a thorough evaluation of the aspects mentioned above in all stages of the infection combined with future studies concentrating on body composition with cardiopulmonary and motor control (gait and balance) rehabilitation.

Conflicts of interest/Competing interests: Authors report no conflict or competing interest.

\section{Reference}

1. Centers for Disease Control and Prevention. (2019). Estimated HIV incidence and prevalence in the United States, 2010-2016. HIV Surveillance Supplemental Report, 24(No. 1).

2. Banks, L.M., Zuurmond, M., Ferrand, R., \& Kuper, H. (2015). The relationship between HIV and prevalence of disabilities in sub-Saharan Africa: A systematic review. Tropical Medicine and International Health. 20(4), 411-429. doi: 10.1111/tmi.12449.

3. Erlandson, K.M., Allshouse, A.A., Jankowski, CM, et al. (2012). Comparison of Functional Status Instruments in HIV-Infected Adults on Effective Antiretroviral Therapy. HIV Clinical Trials. 13(6):324-334. doi:10.1310/hct1306-324

4. Cohen, H.S., Cox, C., Springer, G, et al. (2012). Prevalence of abnormalities in vestibular function and balance among HIVseropositive and HIV-seronegative women and men. PLoS One. 7(5):e38419. doi:10.1371/journal.pone.0038419

5. Heinze, B., Swanepoel, D.W., \& Hofmeyr, L.M. (2011). Systematic review of vestibular disorders related to human immunodeficiency virus and acquired immunodeficiency syndrome. The Journal of Laryngology \& Otology. 125(9):881890. doi:10.1017/s0022215111001423.

6. Berner, K., Morris, L., Baumeister, J., \& Louw, Q. (2017). Objective impairments of gait and balance in adults living with HIV-1 infection: a systematic review and meta-analysis of observational studies. BMC Musculoskeletal Disorders. 18(1). doi:10.1186/s12891-017-1682-2.

7. Bauer, L.O., Wu, Z., Wolfson, L.I. (2011). An obese body mass increases the adverse effects of HIV/AIDS on balance and gait. Phys Ther. 91(7):1063-1071. doi:10.2522/ptj.20100292 
8. O'Brien, K. K., Tynan, A. M., Nixon, S. A., \& Glazier, R. H. (2016). Effectiveness of aerobic exercise for adults living with HIV: systematic review and meta-analysis using the Cochrane Collaboration protocol. BMC infectious diseases, 16, 182. https://doi.org/10.1186/s12879-016-1478-2

9. Bauer L. O. (2018). HIV/AIDS and an overweight body mass are associated with excessive intra-individual variability in response preparation. Journal of neurovirology, 24(5), 577-586. https://doi.org/10.1007/s13365-018-0644-2

10. MacArthur, R.D., Levine, S.D., \& Birk, T.J. (1993). Supervised exercise training improves cardiopulmonary fitness in HIVinfected persons. Med Sci Sports Exerc. 25:684-8.

11. Rosario, M.G., Jamison L., \& Gines G. (2020). The Role of HIV Antiretroviral Medication on Motor-Cognitive and Neurological Alterations in Hispanic People Living with HIV. J Pub Health Issue Pract 4(1):160. doi: https://doi.org/10.33790/ jphip1100160

12. Terry, L., Sprinz, E., \& Ribeiro, J.P. (1999). Moderate and high intensity exercise training in HIV-1 seropositive individuals: a randomized trial. Int J Sports Med. 20:142-6.

13. Tiozzo, Eduard, (2011). "The Effect of Combined ModerateIntensity Training on Immune Functioning, Metabolic Variables, and Quality of Life in HIV-infected Individuals Receiving Highly Active Antiretroviral Therapy". Open Access Dissertations. 678.

14. Perez-Moreno, F., Camara-Sanchez, M., Tremblay, J.F., RieraRubio, V.J., Gil-Paisan, L., \& Lucia, A. (2007). Benefits of exercise training in Spanish prison inmates. Int J Sports Med. 28:1046-52.

15. Mutimura, E., Stewart, A., Crowther, N.J., Yarasheski, K.E., \& Cade, W.T. (2008). The effects of exercise training on quality of life in HAART-treated HIV-positive Rwandan subjects with body fat redistribution. Qual Life Res. 17:377-85.

16. Baigis, J., Korniewicz, D.M., Chase, G., Butz, A., Jacobson, D., \& Wu, A.W. (2002). Effectiveness of a home-based exercise intervention for HIV-infected adults: a randomized trial. J Assoc Nurses AIDS Care. 13:33-45.

17. Maharaj, S.S., Chetty, V. (2011). Rehabilitation program for the quality of life for individuals on highly active antiretroviral therapy in KwaZulu-Natal, South Africa: a short report. Int J Rehabil Res. 34:360-5

18. LaPerriere, A., Fletcher, M.A., Antoni, M.H., Klimas, N.G., Ironson, G., \& Schneiderman, N. (1991). Aerobic exercise training in an AIDS risk group. Int J Sports Med. 12 Suppl $1:$ S53-7

19. Lox, C.L., McAuley, E., \& Tucker, R.S. (1996). Aerobic and resistance exercise training effects on body composition, muscular strength, and cardiovascular fitness in an HIV-1 population. Int J Behav Med. 3:55-69.

20. Balasubramanyam, A., Coraza, I., Smith, E.O., Scott, L.W., Patel, P., \& Iyer D, et al. (2011). Combination of niacin and fenofibrate with lifestyle changes improves dyslipidemia and hypoadiponectinemia in HIV patients on antiretroviral therapy: results of "heart positive," a randomized, controlled trial. J Clin Endocrinol Metab. 96:2236-47.

21. Ogalha, C., Luz, E., Sampaio, E., Souza, R., Zarife, A., Neto MG, et al. (2011). A randomized, clinical trial to evaluate the impact of regular physical activity on the quality of life, body morphology and metabolic parameters of patients with AIDS in Salvador, Brazil. J Acquir Immune Defic Syndr. 57 Suppl 3:S179-85.
22. Farinatti, P.T., Borges, J.P., Gomes, R.D., Lima, D., \& Fleck, S.J. (2010). Effects of a supervised exercise program on the physical fitness and immunological function of HIV-infected patients. J Sports Med Phys Fitness. 50:511-8.

23. Vajpayee, M., Kaushik, S., Sreenivas, V., Wig, N., \& Seth, P. (2005). CdC staging based on absolute Cd4 count and Cd4 percentage in an HIV-1-infected Indian population: treatment implications. Clinical and experimental immunology, 141(3), 485-490. https://doi.org/10.1111/j.1365-2249.2005.02857.x

24. Lox, C.L., McAuley, E., \& Tucker, R.S. (1996). Aerobic and resistance exercise training effects on body composition, muscular strength, and cardiovascular fitness in an HIV-1 population. Int J Behav Med. 3:55-69.

25. Oursler, K.K.; O'Boyle, H.M., Briggs, B.C., Sorkin, J.D., Jarmukli, N., Katzel, L.I., Freiberg, M.S., \& Ryan, A.S. (2019). Association of Diastolic Dysfunction with Reduced Cardiorespiratory Fitness in Adults Living with HIV. AIDS Patient Care STDS, vol. 33(12) pp. 493-499

26. Erqou, S., Lodebo, B.T., Masri, A., Altibi, A.M., EchouffoTcheugui, J.B., Dzudie, A., Ataklte, F., Choudhary, G., Bloomfield, G.S., Wu, W.C.1., \& Kengne, A.P. (2019). Cardiac Dysfunction Among People Living With HIV: A Systematic Review and Meta-Analysis.JACC Heart Fail. 7(2):98-108. doi: 10.1016/j.jchf.2018.10.006

27. Rosario, M.G and Gonzalez-Sola, M. (2018). Autonomic nervous system assessment in people with HIV: A cross-sectional study [version 1; peer review: 1 not approved]. F1000Research, 7:696 (https://doi.org/10.12688/f1000research.14685.1)

28. Boldt, A. (2019). What Incline on a Treadmill Is the Same As a Flat Surface? SportsRec.

29. Rosario, M.G., Marshall, J., Herkert, A., Binoy, B., Windham, H., \& Orozco, E. (2020). Lower Limb Neuromuscular Modification and Standing Postural Control Alteration in Apparent Asymptomatic People Living with HIV. J Rehab Pract Res 1(1):102. https://doi.org/10.33790/jrpr1100102

30. Calza, L., Manfredi, R., \& Chiodo, F. (2004). Dyslipidaemia associated with antiretroviral therapy in HIV-infected patients.J Antimicrob Chemother. 53(1):10-4. Epub 2003 Nov 25.

31. Calza, L., Manfredi, R., Pocaterra, D., \& Chiodo, F. (2008). Risk of premature atherosclerosis and ischemic heart disease associated with HIV infection and antiretroviral therapy. J Infect. 57(1):16-32. doi: 10.1016/j.jinf.2008.02.006. Epub 2008 Mar 21.

32. Trenkwalder, C., Straube, A., Paulus, W., Krafczyk, S., Schielke, E., \& Einhäupl, K. (1992). Postural imbalance: An early sign in HIV-1 infected patients. European Archives of Psychiatry and Clinical Neuroscience., 241(5), 267-272.

33. Falusi, O.M., Aberg, J.A. (2001). HIV and cardiovascular risk factors. AIDS Read, vol. 11(5) pp. 263-8

34. Sabin, C. A. (2013). Do people with HIV infection have a normal life expectancy in the era of combination antiretroviral therapy?. BMC medicine, 11, 251. https://doi.org/10.1186/1741-7015-11251

35. Jia, H., Zack, M.M., Gottesman, II., Thompson, W.W. (2018). Associations of Smoking, Physical Inactivity, Heavy Drinking, and Obesity with Quality-Adjusted Life Expectancy among US Adults with Depression. Value Health. 21(3):364-371. doi: 10.1016/j.jval.2017.08.002. Epub 2017 Oct 18. 